gr-qc/9406045

QMW-Maths-1994-SPB-1 preprint June 21, 2017

\title{
Hypertime Formalism for Spherically Symmetric Black Holes and Wormholes
}

\author{
Stephen P. Braham \\ School of Mathematical Sciences \\ Queen Mary and Westfield College, University of London \\ Mile End Road. London E1 $4 N S$ \\ United Kingdom \\ E-mail: S.P.Braham@qmw.ac.uk
}

\begin{abstract}
Recent work on an approach to the geometrodynamics of cylindrical gravity waves in the presence of interacting scalar matter fields, based on the Kuchař hypertime formalism, is extended to the analogous spherically symmetric system. This produces a geometrodynamical formalism for spherical black holes and wormholes in which the metric variables are divided into two classes, dynamical and redundant. The redundant variables measure the embedding of a spacelike hypersurface into the spacetime, and proper time in the asymptotically flat regions. All the constraints can be explicitly solved for the momenta conjugate to the embedding variables. The dynamical variables, including an extra ADM mass for wormhole topologies, can then be considered as functionals of the redundant ones, including the proper time variable. The solution of the resulting constraint system determines the momentum conjugate to the proper time as a function of the other variables, producing Unruh's Hamiltonian formalism for the spherical black hole, whilst extending it to an arbitrary foliation choice. The resulting formalism is appropriate as a starting point for the construction of a hypertime functional Schrödinger equation for quantized spherically symmetric black holes and wormholes.
\end{abstract}




\section{Introduction}

Classical and quantum geometrodynamics (CGD and QGD) are haunted, and made more interesting, by the problem of time. This is simply the problem that general relativity, through its general covariance, does not possess a special, external, time parameter, and thus it is difficult to understand the theory in terms of dynamics. In the classical case, it is possible to avoid this problem if we view gravity through the canonical formalism first set up by Dirac [1], as well as by Arnowitt, Deser and Misner (ADM) [2]. In this formalism, the spacetime 4-metric is decomposed in terms of the familiar 3-metric $g_{i j}$ and the corresponding lapse $N$, and shift $N^{i}$ variables, all introduced on a specific foliation (prescribed by $N$ and $N^{i}$ ), labeled by a time parameter $t$, and a spatial hypersurface position $\mathbf{x}$. The action for the vacuum part of the theory can then be written in the manifestly canonical form

$$
\left.S=\int d t \int d^{3} x\left(\pi^{i j}(\mathbf{x}) \dot{g}_{i j}(\mathbf{x})-N(\mathbf{x}) \mathcal{H}(\mathbf{x})-N_{i}(\mathbf{x}) \mathcal{H}^{(} \mathbf{x}\right)\right)
$$

with the overdot representing partial differentiation with respect to $t$, and $\pi^{i j}$ being the momenta conjugate to $g_{i j}$. This allows us to view the theory as describing the evolving 3geometry (and corresponding momenta) on a hypersurface, but under the superhamiltonian and supermomentum constraints

$$
\mathcal{H}(\mathbf{x})=0, \mathcal{H}^{i}(\mathbf{x})=0
$$

respectively, that are generated by variation of $N$ and $N_{i}$ in Equation (11). These constraints contain all the information to evolve the system, given the lapse and shift [2]. In this sense, we can impose a concept of time on the theory. However, even in the classical case, this position is a little uncomfortable, as we are forced to consider each possible foliation separately, and, furthermore, there is generally no geometric way of identifying a choice of $t$. In the quantum theory, things are even worse. In this case, the quantum equations describing the system are given by Equation (2), under the usual canonical quantization rules for replacing phase-space variables with operators,

$$
g_{i j} \rightarrow \hat{g}_{i j}=g_{i j} \times, \pi^{i j} \rightarrow \hat{\pi}^{i j}=-i \frac{\delta}{\delta g_{i j}},
$$

operating on some wavefunction $\psi\left[g_{i j}\right]$. These equations, as they stand, cannot be viewed, in any way, as describing the evolution of a quantum state with respect to $t$ and, in fact, describe a state that is manifestly independent of $t$. So, our "fix" does not extend to the quantum theory.

One possible solution to the problem of time is to view the constraints given by Equation (2) as representing evolution with respect to other variables, constructed out of the gravitational and matter phase-space variables themselves, instead of with respect to the simple label $t$. The latter are called embedding variables [3, [, 5] and they can be considered as representing the location of a given spacelike hypersurface in an embedding into a surrounding spacetime, thus leading to the term hypertime for such formalisms. In a recent 
paper [6], we looked at this method for the case of cylindrically symmetric spacetimes, and succeeded in constructing hypertime variables for those models. We will be extending that work in this paper to the physically more interesting case of spherically symmetric black holes with self-interacting matter fields.

We proceed as follows: In Section 2 we will briefly describe the hypertime formalism, in the case when we have boundary conditions. In particular we will indicate the conditions needed for a hypertime formalism to be consistent. In Section 3 we will describe the model being used, and construct the corresponding ADM geometrodynamics. The important part of the calculation is then found in Section 1 , in which we actually find a set of hypertime variables for spherically symmetric gravity, and then verify that they meet the conditions described in Section 2. Finally, in Section 5, we will briefly discuss a few points that arise out of such a calculation, including quantization, the connection to work by Unruh [7] and to the work on the semiclassical stability of the inner horizon of black holes, by Hiscock [8] and others.

\section{Hypertime and Boundary Conditions}

We will first need to extend the hypertime formalism developed by Kuchař [9], which is originally applicable only to geometrodynamics without boundary conditions, to one which can handle such conditions. The modifications are, in fact trivial, and we shall just sketch them. The method starts by finding a canonical transformation in which we can divide the standard local gravitational phase-space variables into three classes [9]

$$
g_{i j}, \pi^{i j} \rightarrow T^{\mu}, \Pi_{\mu}, Y_{\mu} \equiv\left\{g^{A}, \pi_{A}\right\},
$$

where $\mu$ ranges from one to four and $A \in\{1,2\} \cdot g^{A}$ and $\pi_{A}$ represent the 'true' dynamical variables of the theory discussed above, which can easily be extended to include matter degrees of freedom without changing our results. $T^{\mu}$ represent the corresponding internal embedding coordinates (describing the location of the hypersurface in spacetime) with $\Pi_{\mu}$ being the corresponding momenta (i.e. the energy-momentum densities). The superhamiltonian and supermomenta will then generally become non-local functionals of the new variables. For geometrodynamics with boundary conditions, we will also allow the hypertime variables to include an added discrete number of boundary hypertime variables $T_{(\partial)}^{\omega}$,

with corresponding momenta $\Pi_{(\partial) \sigma}$, on top of each spatial point hypertime $T^{\mu}(\mathbf{x})$. These will usually be needed when non-vanishing boundary terms are converted to constraints via the method of parametrization of infinity, an example of which is in a recent paper [10] by Kuchař on the geometrodynamics of Schwarzschild wormholes. We will therefore be interested in a total Hamiltonian of the following form:

$$
\mathbf{H}=\int d^{3} x\left(N(\mathbf{x}) \mathcal{H}(\mathbf{x})+N_{i}(\mathbf{x}) \mathcal{H}^{i}(\mathbf{x})\right)+N_{(\partial)} \mathcal{H}_{(\partial)}+N_{i(\partial)} \mathcal{H}_{(\partial)}^{i},
$$

where the Lagrange multipliers $N_{(\partial)}$ and $N_{(\partial)}^{i}$ enforce extra "boundary" constraints,

$$
\mathcal{H}_{(\partial)}\left[T^{\mu}, \Pi_{\nu}, T_{(\partial)}^{\omega}, \Pi_{(\partial) \sigma}, Y_{\rho}\right]=0, \mathcal{H}_{(\partial) i}\left[T^{\mu}, \Pi_{\nu}, T_{(\partial)}^{\omega}, \Pi_{(\partial) \sigma}, Y_{\rho}\right]=0
$$


We can let these constraints be arbitrary functionals of the hypertime and dynamical phasespace variables (as they will usually be generated by non-local canonical transformations acting on standard ADM boundary terms). We further assume that the extra constraints have been chosen such that they are preserved under evolution using the total Hamiltonian, so the extended constraint algebra does not generate any extra constraints. To simplify the equations, and to make the similarity of the calculation to that by Kuchar [9] obvious, we will introduce a special indexing and summation convention. Upper case Greek characters will range over tensor indices, spatial location, and boundary terms, with summation being over the entire range, so that

$$
\Pi_{\Lambda} \dot{T}^{\Lambda}=\int d^{3} x \Pi_{\mu}(\mathbf{x}) \dot{T}^{\mu}(\mathbf{x})+\Pi_{(\partial) \mu} \dot{T}_{(\partial)}^{\mu},
$$

which generalizes the convention used by Kuchař to include the boundary terms. Functional derivative language will be used, with the assumption that we use partial derivatives when operating on appropriate objects. We will also denote time-space pairs of variables $\left[N, N_{i}\right]$ by 4 -vector notation $N_{\mu}$.

With the above notation, we start off by writing down a solution, for $\Pi_{\Lambda}$, to the constraint system,

$$
\Pi_{\Lambda}=-P_{\Lambda}\left[T^{\Sigma}, Y_{\mu}\right]
$$

so that we have

$$
\mathcal{H}^{\Lambda}\left[T^{\Sigma},-P_{\Sigma}\left[T^{\Delta}, Y_{\nu}\right], Y_{\mu}\right]=0 .
$$

Functional differentiation of this gives us the following relations:

$$
\frac{\delta \mathcal{H}^{\Lambda}}{\delta T^{\Sigma}}=\mathcal{H}^{\Lambda \Delta} \frac{\delta P_{\Delta}}{\delta T^{\Sigma}}, \frac{\delta \mathcal{H}^{\Lambda}}{\delta Y^{\mu}}=\mathcal{H}^{\Lambda \Delta} \frac{\delta P_{\Delta}}{\delta Y^{\mu}}
$$

where we define

$$
\mathcal{H}^{\Sigma \Lambda}=\frac{\delta \mathcal{H}^{\Sigma}}{\delta \Pi_{\Lambda}} .
$$

The dynamics of the embedding variables are then given by

$$
\dot{T}^{\Lambda}=N_{\Sigma} \mathcal{H}^{\Sigma \Lambda}
$$

The important point is to now assume that $\mathcal{H}^{\Sigma \Lambda}$ can be inverted, so that we can use Equation (12) to write the lapse-shift vectors $N_{\Sigma}$ in terms of the hypertime velocities $\dot{T}^{\Lambda}$. This is a crucial condition, and signifies the fact that the $T^{\Lambda}$ variables do indeed measure the embedding of the spacelike hypersurface into the surrounding spacetime.

It is then a simple matter to follow Kuchar [9], and substitute the solution for $N_{\Sigma}$ into the Hamilton equations for the dynamical variables

$$
\dot{g}^{A}(\mathbf{x})=N_{\Lambda} \frac{\delta \mathcal{H}^{\Lambda}}{\delta \pi_{A}(\mathbf{x})}, \dot{\pi}_{A}(\mathbf{x})=-N_{\Lambda} \frac{\delta \mathcal{H}^{\Lambda}}{\delta g^{A}(\mathbf{x})},
$$


to get the following evolution equation:

$$
\dot{Y}^{\mu}=\left[Y^{\mu}, P_{\Sigma}\right]_{P} \dot{T}^{\Sigma}
$$

where $[,]_{P}$ denotes the Poisson bracket over just the $Y^{\mu}$ variables. The central point of the hypertime approach is to assume that $Y^{\mu}$ can be considered to be a functional of the total hypertime $T^{\Lambda}$ that specifies the position of a spacelike hypersurface, and not of the label time $t$. The evolution of $Y^{\mu}$ between one hypersurface 'at' hypertime $T_{1}^{\Lambda}$ and another one at hypertime $T_{2}^{\Lambda}$ should therefore be independent of the hypertime foliation path $T^{\Lambda}(t)$ between them. This leads to the following hypertime functional Hamilton equation:

$$
\frac{\delta Y_{\mu}}{\delta T^{\Lambda}}=\left[Y_{\mu}, P_{\Lambda}\right]_{P}
$$

For the evolution to actually be independent of the path, we need to sure that the functional derivatives that are defined the above equation weakly $(\approx)$ commute, i.e.

$$
\frac{\delta}{\delta T^{\Sigma}}\left[Y_{\mu}, P_{\Lambda}\right]_{P}=\frac{\delta}{\delta T^{\Lambda}}\left[Y_{\mu}, P_{\Sigma}\right]_{P}
$$

when the constraints are satisfied. It is then simple to show that this is equivalent to the following condition:

$$
\frac{\delta P_{\Lambda}}{\delta T_{\Sigma}}-\frac{\delta P_{\Sigma}}{\delta T_{\Lambda}}+\left[P_{\Lambda}, P_{\Sigma}\right]_{P}=0
$$

which is the extension of the equivalent condition discussed by Kuchař [9] that one would naively expect. It is a simple matter [9] to show that this is directly implied by the (weak) vanishing of the total constraint algebra

$$
\left[\mathcal{H}^{\Lambda}, \mathcal{H}^{\Sigma}\right]_{T} \approx 0
$$

by using the assumed invertability of $\mathcal{H}^{\Lambda \Sigma}$ (where the bracket $[,]_{T}$ is over all variables). Indeed, all we need to do is use the inverse of $\mathcal{H}^{\Lambda \Sigma}$, and the relations given in Equation (10) to rewrite the commutator in terms of $P_{\Lambda}$, giving Equation (17). Thus it is easy to absorb boundary constraints into the hypertime formalism, and to arrive at the generalized hypertime evolution equation given by Equation (15).

The structure of the boundary terms in Equation (5) is very important. If, instead of constraints at the boundary, we had non-vanishing boundary contributions to the total Hamiltonian, we would not have the simple equivalence between our formalism and that of Kuchař. In particular, the inversion of Equation (12) would be more complicated, and the consistency of Equation (15) would no longer be guaranteed by the constraint algebra closure condition, Equation (18).

So, we have reduced finding a consistent geometrodynamical formulation down to finding $P_{\Lambda}$. This may, in general, be impossible for full general relativity, but it still seems useful to investigate the hypertime formalism for model systems with extra symmetry. Just such a case was investigated in Ref. [6], when no extra boundary terms were needed to achieve the correct dynamics. Now we are ready to reduce spherically symmetric gravity to a Hamiltonian of the form given by Equation (5), and construct the corresponding $P_{\Lambda}$ functionals. 


\section{Spherically Symmetric Geometrodynamics}

We will be interested in a charged spherically symmetric metric minimally coupled to a set of interacting real scalar fields. The charge will allow for Reissner-Nordström wormhole solutions as well as the more usual black hole ones. The scalar fields will emulate the important technical aspects of a general matter content, without adding the complexities of extra gauge constraints. The correct geometrodynamics of such models were first formulated by Unruh [0], based on a paper by Berger, Chitre, Moncrief and Nutku [11]. The model has been investigated in depth by Hajicek et al [12, 13, 14, 15]. Recently the vacuum geometrodynamics for spherical black holes have been investigated by D. Louis-Martinez, J. Gegenberg and G. Kunstatter [16], with a corresponding hypertime formulation in the paper by Kuchař [10].

We will write the spherically symmetric metric, with charge $q$, in a form that will lead to an action that mirrors the one for cylindrically symmetric metrics used in Ref. [6]

$$
d s^{2}=\varphi^{-\frac{1}{2}} e^{2 \gamma}\left(\left(-\alpha^{2}+\beta^{2}\right) d t^{2}+2 \beta d t d t+d r^{2}\right)+\varphi\left(d \theta^{2}+\sin ^{2}(\theta) d \phi^{2}\right),
$$

where the functions $\varphi, \gamma, \alpha$ and $\beta$ are functions only of the time $t$ and radial coordinate $r$, and not of the coordinates for the surfaces of spherical symmetry $\theta$ and $\phi$. Each 2sphere has area $4 \pi \phi(t, r)$, and $\gamma(t, r)$ specifies a conformal factor on a 2-D spacetime with coordinate $(t, r)$ and metric

$$
{ }^{(2)} d s^{2}=e^{2 \gamma}\left(\left(-\alpha^{2}+\beta^{2}\right) d t^{2}+2 \beta d t d t+d r^{2}\right) .
$$

The functions $\alpha(t, r)$ and $\beta(t, r)$ are the lapse and shift functions, respectively, connecting one constant $t$ spacelike hypersurface to another. We now couple the metric to $N$ real scalar fields, $f_{(i)}$, with an arbitrary self-interaction potential $V\left(f_{(i)}\right)$, giving an action in the 2-covariant form

$$
S=-\int d t d r \sqrt{g}\left(\frac{1}{4} \varphi R+\varphi \sum_{i=0}^{N} \nabla^{a} f_{(i)} \nabla_{a} f_{(i)}+\varphi^{\frac{1}{2}} V+\frac{1}{2} q^{2} \varphi^{-\frac{3}{2}}-\frac{1}{2} \varphi^{-\frac{1}{2}}\right),
$$

where $g, R$, and $\nabla_{a}$ represent the metric determinant, scalar curvature and covariant derivative respectively on the 2-D spacetime with metric given by Equation (20). There will be important boundary corrections to this action that we will calculate later.

We will assume that the space is asymptotically flat in either one or two regions, and that $(t, \pm r)$ are the standard Minkowski coordinates in the respective asymptotic limits. If there is only one asymptotic region $r \rightarrow \infty$, then we will have a regular centre at $r=0$, and the spacelike hypersurfaces will have topology $\mathbf{R}^{3}$. If we have two asymptotic regions $r \rightarrow \pm \infty$, then we will have a wormhole topology, and the spacelike hypersurfaces will be 3-cylinders $\left(\mathbf{R} \times \mathbf{S}^{2}\right)$. In either case, $\varphi$ tends asymptotically to $r^{2}$ for large $|r|$, with corrsponding conditions on $\gamma$. For the matter fields, we will assume that $f_{(i)}=o\left(|r|^{-1 / 2}\right)$, and that $V=o\left(|r|^{-2}\right)$ for $|r| \rightarrow \infty$. 
We can now write down the standard ADM [2] formulation of the model, with $t$ acting as foliation label. The total action, up to boundary terms, takes the following form:

$$
S=\int d t d r\left(\pi_{\gamma} \dot{\gamma}+\pi_{\varphi} \dot{\varphi}+\sum_{i=0}^{N} \pi_{(i)} \dot{f}_{(i)}-\alpha \mathcal{H}-\beta \mathcal{H}^{\prime}\right),
$$

where $\pi_{\gamma}, \pi_{\varphi}$ and $\pi_{(i)}$ are the momenta conjugate to $\gamma, \varphi$ and $f_{(i)}$ respectively. $\mathcal{H}$ and $\mathcal{H}^{\prime}$ are the superhamiltonian,

$$
\mathcal{H}=-2 \pi_{\gamma} \pi_{\varphi}-\frac{1}{2} \gamma_{, r} \varphi_{, r}+\frac{1}{2} \varphi_{, r r}+e^{2 \gamma}\left(\varphi^{2} V+\frac{1}{2} q^{2} \varphi^{-\frac{3}{2}}-\frac{1}{2} \varphi^{\frac{1}{2}}\right)+\mathcal{H}_{f}
$$

and supermomentum

$$
\mathcal{H}^{\prime}=\pi_{\gamma} \gamma_{, r}+\pi_{\varphi} \varphi_{, r}-\pi_{\gamma, r}+\mathcal{H}_{f}^{\prime},
$$

and we have written $\mathcal{H}_{f}$ and $\mathcal{H}_{f}^{\prime}$ for the $V=0$ part of the superhamiltonian and supermomentum contributions for the scalar fields. The momenta take the simple form

$$
\begin{gathered}
\pi_{\gamma}=-\frac{1}{2 \alpha}\left(\dot{\varphi}-\beta \varphi_{, r}\right), \\
\pi_{\varphi}=-\frac{1}{2 \alpha}\left(\dot{\gamma}-\beta_{, r}-\beta \gamma_{, r}\right), \\
\pi_{(i)}=-\frac{2 \varphi}{\alpha}\left(\dot{f}_{(i)}-\beta f_{(i), r}\right) .
\end{gathered}
$$

The resulting constraints and momenta are very closely related to those found for the cylindrically symmetric system discussed in Ref. [6].

\section{Hypertime for Black Holes}

Now we are ready to take the results of Sections 2 and 3 and formulate a hypertime geometrodynamics for black holes and wormholes. We are directly motivated by Ref. [6], and therefore use basically the same hypertime variable (which is derived from those originally discovered by Kuchař for vacuum cylindrically symmetric spacetimes [17]). We define

$$
T^{ \pm}(r)=-2 \int_{r_{L}}^{r} \pi_{\gamma}\left(r^{\prime}\right) d r^{\prime} \pm \varphi
$$

with $r_{L}$ being the corresponding leftmost $r$ for the wormhole and regular centre topologies. The corresponding momenta are given by

$$
\Pi_{ \pm}=\frac{1}{4} \frac{\partial}{\partial r} \ln \left( \pm e^{-\gamma} \frac{\partial T^{ \pm}}{\partial r}\right) \pm \frac{1}{2} \pi_{\varphi}
$$

These variables basically measure the rate of growth of the area function $\varphi$ along appropriately parametrized outgoing (increasing $r$ ) and incoming (decreasing $r$ ) null geodesics, 
integrated along the spacelike hypersurface. Indeed, Equation (25) can be used to show that an outer apparent horizon occurs at $T_{, r}^{+}=0$, and an inner one at $T_{, r}^{-}=0$, where our sense of outer and inner is respect to the rightmost asymptotically flat region. For a smooth, non-singular foliation, the hypertime variables themselves are continuous and finite across these horizons, and their momenta are well-defined on either size of each horizon.

It is straightforward to show that

$$
\left(f_{(i)}, \pi_{(i)}, \gamma, \varphi, \pi_{\gamma}, \pi_{\varphi}\right) \rightarrow\left(f_{(i)}, \pi_{(i)}, T^{ \pm}, \Pi_{ \pm}\right)
$$

is a canonical transformation but, unlike the same transformation in Ref. [6], it is not enough, on its own, to form a hypertime transformation. The crucial missing factor is the importance of the ADM boundary term in the geometrodynamics of black holes and wormholes. We can see this immediately, if we consider the paper by Unruh [7]. In that paper, a specific foliation and gauge choice was used, corresponding to $\pi_{\gamma}=0$ and $\varphi=r^{2}$ respectively. However, that choice corresponds to $\dot{T}^{ \pm}(r)=0$, and therefore we need an extra hypertime variable to track the evolving position of each leaf of the foliation, as $t$ increases into the future. From our discussion in Section 2 we can easily guess that the extra variable will be a boundary hypertime.

To construct the appropriate boundary terms, we will first write down the transformed versions of Equations (23) and (24).

$$
\begin{gathered}
\mathcal{H}=\Pi^{+} T_{, r}^{+}-\Pi^{-} T_{, r}^{-}+\mathcal{H}_{f}-\frac{1}{8}\left(2 V+\frac{q^{2}}{\varphi^{2}}-\frac{1}{\varphi}\right) T_{, r}^{+} T_{, r}^{-} \exp \left(\int_{r}^{\infty} d r^{\prime}\left(4 \Pi^{+}+4 \Pi^{-}-\frac{1}{2 \varphi} \frac{\partial \varphi}{\partial r}\right)\right) \\
\mathcal{H}^{\prime}=\Pi^{+} T_{, r}^{+}+\Pi^{-} T_{, r}^{-}+\mathcal{H}_{f}^{\prime}
\end{gathered}
$$

with $\varphi$ now simply being defined by Equation (28). However, if we smear these with the lapse and shift, to get the total Hamiltonian, and vary, we get the following remaining variations at each boundary:

$$
\delta \int d r^{\prime}\left(\alpha \mathcal{H}+\beta \mathcal{H}^{\prime}\right)=-\frac{1}{2} \delta \rho
$$

where $\rho=\sqrt{|\varphi|}$, and we have solved the variation equations everywhere else. This produces no extra variation at a regular centre $\rho=0$, but we will need a correction term at each asymptotic infinity. We find that we can cancel these variations by adding the following correction to the total Hamiltonian at each infinite limit of the $r$ integration:

$$
\alpha_{ \pm} H_{B \pm}=\alpha_{ \pm} \lim _{r \rightarrow \pm \infty} \frac{1}{2} \rho \int_{r}^{\infty} d r^{\prime}\left(4 \Pi^{+}+4 \Pi^{-}-\frac{1}{\rho} \frac{\partial \rho}{\partial r}\right)
$$

where $\alpha \rightarrow \alpha_{ \pm}$as $r \rightarrow \pm \infty$. We can understand these terms if we look at the effective ADM mass at radius $r$. The latter, scalar invariant, quantity can be defined by using the fact that we can always find a coordinate system $(\bar{t}(t, r), \bar{r}(t, r))$ for a spherically symmetric 
geometry, at a single point, in which the metric becomes that of a Reissner-Nordström spacetime, with local effective ADM mass $m(r)$ and charge $q$,

$$
d s^{2}=-\left(1-\frac{2 m(r)}{\bar{r}}+\frac{q^{2}}{\bar{r}^{2}}\right) d \bar{t}^{2}+\left(1-\frac{2 m(r)}{\bar{r}}+\frac{q^{2}}{\bar{r}^{2}}\right)^{-1} d \bar{r}^{2}+\bar{r}^{2}\left(d \theta^{2}+\sin ^{2}(\theta) d \phi^{2}\right) .
$$

If we calculate $\gamma$ by inverting Equation (29), then we end up with the following very important equation:

$$
\Pi^{+}+\Pi^{-}=\frac{1}{4} \frac{\partial}{\partial r} \ln \left(\rho-2 m+\frac{q^{2}}{\rho}\right)
$$

We can now use this relationship to get

$$
H_{B \pm}=\lim _{r \rightarrow \pm \infty} m(r)
$$

by simple substitution in Equation (34). Thus $H_{B \pm}$ represents the standard ADM mass correction, well known for spherical symmetry [7, 12], simply rewritten in terms of the new variables. The complete total Hamiltonian will reproduce the equations of motion, but subject to the condition that $\alpha$ is fixed at $r= \pm \infty$. This is good enough for normal geometrodynamics but is not good enough for hypertime geometrodynamics, from Section 2, and specifically for the following reason: To arrive at consistent hypertime dynamics, the boundary terms must be constrained to vanish, which would force $H_{B \pm}=0$ (corresponding to allowing variation of $\alpha$ at infinity), and thus force the vanishing of the ADM mass at each infinity. The latter obviously cannot be allowed to happen if we wish to study generic, non-vacuum, solutions. Kuchař, however, has already addressed this problem in Ref. [10], and indicated that we can use the so-called method of parametrization of infinities to transform our boundary terms to valid boundary constraints.

In our case, the method of parametrization of infinities starts by defining two new variables, suggestively named $\tau_{ \pm}$, with corresponding momenta $\pi_{B \pm}$, and then using the following, extended, total Hamiltonian:

$$
\mathbf{H}=\int d r^{\prime}\left(\alpha \mathcal{H}+\beta \mathcal{H}^{\prime}\right)+\alpha_{+}\left(\pi_{B+}+H_{B+}\right)+\alpha_{-}\left(\pi_{B-}+H_{B-}\right),
$$

where the final term is discarded when we have a regular centre. This then gives us the following equations of motion for the $\tau_{ \pm}$variables:

$$
\dot{\tau}_{ \pm}=\alpha_{ \pm}=\lim _{r \rightarrow \pm \infty} \alpha(r)
$$

and thus, on solutions, $\tau_{ \pm}$are just the proper times at each infinity. We can now leave $\alpha$ free to vary, even at infinity. We have the basic formalism described in Section 2, except that we still need to identify a boundary hypertime variable, or variables. Note that the total constraint system is consistent, due to the fact that $\mathbf{H}$ does not contain $\tau^{ \pm}$, and so $\pi_{B \pm}$ are constants of the motion, as are the ADM masses. 
Will we first find the general solution to the local constraints, i.e. to

$$
\mathcal{H}\left[T^{ \pm}, \Pi_{ \pm}, f_{(i)}, \pi_{(i)}\right]=0, \mathcal{H}^{\prime}\left[T^{ \pm}, \Pi_{ \pm}, f_{(i)}, \pi_{(i)}\right]=0 .
$$

If we use the effective ADM mass $m(r)$, via Equation (36), we can use the constraints to eliminate one functional freedom in the solutions, and simplify the problem down to

$$
\begin{gathered}
P_{+}=\frac{T_{, r}^{-}}{4\left(T_{, r}^{+}-T_{, r}^{-}\right)} \frac{\partial}{\partial r} \ln \left(\rho-2 m+\frac{q^{2}}{\rho}\right)+\frac{\mathcal{H}_{f}^{\prime}}{\left(T_{, r}^{+}-T_{, r}^{-}\right)}, \\
P_{-}=-\frac{T_{, r}^{+}}{4\left(T_{, r}^{+}-T_{, r}^{-}\right)} \frac{\partial}{\partial r} \ln \left(\rho-2 m+\frac{q^{2}}{\rho}\right)-\frac{\mathcal{H}_{f}^{\prime}}{\left(T_{, r}^{+}-T_{, r}^{-}\right)},
\end{gathered}
$$

Where $P_{ \pm}$are the objects defined in Section 2. We can now rewrite the remaining part of the constraints in terms of $m(r)$, giving

$$
\frac{\partial}{\partial r}\left(\rho-2 m+\frac{q^{2}}{\rho}\right)+2 h_{f}\left(\rho-2 m+\frac{q^{2}}{\rho}\right)+\rho_{, r}\left(2 V \rho^{2}+\frac{q^{2}}{\rho^{2}}-1\right)=0,
$$

where we have

$$
h_{f}=\frac{1}{T_{, r}^{+}}\left(\mathcal{H}_{f}+\mathcal{H}_{f}^{\prime}\right)-\frac{1}{T_{, r}^{-}}\left(\mathcal{H}_{f}-\mathcal{H}_{f}^{\prime}\right) .
$$

It is a simple matter to solve this equation to get

$$
m(r)=\exp \left(-2 \int_{r_{L}}^{r} d r h_{f}\right) M_{L}+\int_{r_{L}}^{r} d r^{\prime}\left(h_{f}\left(1+\frac{q^{2}}{\rho^{2}}\right)+V \rho \rho_{, r^{\prime}}\right) \rho \exp \left(-2 \int_{r^{\prime}}^{r} d r^{\prime \prime} h_{f}\right),
$$

where $M_{L}=m\left(r_{L}\right)$ is a free integration constant.

We can now demonstrate that one, and only one, of the new variables $\tau_{ \pm}$can always be considered to be redundant, by considering Equation (45) for each topology. For a regular centre, $r_{L}=0$, we must have $M_{L}=0$, and we then have the constraint $\pi_{B+}+H_{B+}=0$, which, after solving Equation (40), corresponds to

$$
\pi_{B+}+\int_{0}^{\infty} d r\left(h_{f}\left(1+\frac{q^{2}}{\rho^{2}}\right)+V \rho \rho_{, r}\right) \rho \exp \left(-2 \int_{r}^{\infty} d r^{\prime} h_{f}\right)=0 .
$$

Thus $\pi_{B+}$ is completely determined, and therefore $\tau_{+}$is a redundant variable. For the wormhole case, $r_{L}=-\infty$, we have two boundary terms. The leftmost constraint, using Equation (45), is simply $\pi_{B-}+M_{L}=0$. This suggests that we make a simple canonical transformation, and define $m_{-}=-\pi_{B-}$, with corresponding momentum $\pi_{m-}=\tau_{-}$. Obviously $m_{-}$is just the ADM mass measured at the left infinity. The leftmost constraint is then just $M_{L}=m_{-}$. Taking this into account, the remaining, rightmost, constraint becomes

$\pi_{B+}+\exp \left(-2 \int_{-\infty}^{\infty} d r h_{f}\right) m_{-}+\int_{-\infty}^{\infty} d r\left(h_{f}\left(1+\frac{q^{2}}{\rho^{2}}\right)+V \rho \rho_{, r}\right) \rho \exp \left(-2 \int_{r}^{\infty} d r^{\prime} h_{f}\right)=0$. 
We therefore find that $\pi_{B+}$ is determined once more, and $\tau_{+}$is again redundant.

The redundancy of $\tau_{+}$suggests that we define

$$
T_{(\partial)}=\tau_{+}, \Pi_{(\partial)}=\pi_{B+},
$$

with $P_{(\partial)}$ being the corresponding solution for $\pi_{B+}$, either from Equation (46) or Equation (47). In the wormhole case, the remaining variable $m_{-}$becomes one of the dynamical variables. This gives us a set of $P_{\Lambda}$ functions, as discussed in Section 2, with a corresponding division of our extended configuration space into dynamical and hypertime variables. All that we need do now is verify that we can invert the Hamilton equations for the hypertime velocities $\dot{T}^{\Lambda}$ to get the lapse and shift, and then the requirements specified in Section 2 will be satisfied. It would a horrendous algebraic problem to find the general solution to Equation (12), using the total Hamiltonian that arises for the spherically symmetric model, but we are lucky, as this is not something we need to do. Continuity of the functional derivatives in $\mathcal{H}^{\Sigma \Lambda}$, with respect to the phase-space variables, ensures that there will exist an inverse, in general, as long as we can find one for a specific choice of those variables. We can simply choose the foliation Unruh foliation and gauge choice described above, and choose a vacuum configuration of the fields. This results in the following equations:

$$
0=2 r \alpha \pm 2 r \beta+2 r \alpha_{+}-2 \int_{0}^{r} d r^{\prime}\left(\alpha+\alpha_{+}\right) .
$$

It is simple to verify that this gives us $\alpha=\alpha_{+}$and $\beta=0$, consistent with the boundary condition at $r=\infty$. Thus the problem has an inverse in flat space and a corresponding general solution. The conditions for consistency of the hypertime formalism, given in Section 2 are now satisfied, and we have achieved the aim of this paper.

\section{Discussion}

The Unruh formalism for spherically symmetric spacetimes [7] provides a valid Hamiltonian geometrodynamics for collapsing matter. That formalism, however, is for a specific choice of time (foliation) and gauge and, up to now, it has been hard to tell whether the manifest breaking of covariance causes any problems. If we can show that it corresponds to one of the 'paths' in our hypertime formalism, then we may feel better about using it as a basis for further (e.g. quantum) work. We also provide a check on the new formalism itself.

The Unruh foliation and gauge corresponds to

$$
\dot{T}^{ \pm}=0, \rho=r, \dot{T}_{(\partial)}=1 .
$$

Equation (14) then tells us that we have

$$
\dot{f}=\left[f, \int_{0}^{\infty} d r\left(h_{f}\left(1+\frac{q^{2}}{r^{2}}\right)+V r\right) r \exp \left(-2 \int_{r}^{\infty} d r^{\prime} h_{f}\right)\right]_{P},
$$


for a single scalar field $f$, with the same equation for the corresponding momentum. Thus we have a total reduced Hamiltonian given by

$$
H=\int_{0}^{\infty} d r\left(h_{f}\left(1+\frac{q^{2}}{r^{2}}\right)+V r\right) r \exp \left(-2 \int_{r}^{\infty} d r^{\prime} h_{f}\right),
$$

which is identical to that given by Unruh [7], once the correct matter fields are specified. Thus that formalism corresponds to a specific path amongst all those possible in the hypertime formalism, all of which produce equivalent dynamics.

This becomes important if we briefly examine the quantization of our formalism. Under the general canonical quantization rules, Equation (8) gives us the hypertime functional Schrödinger equation

$$
i \frac{\delta}{\delta T^{\Lambda}} \psi\left[T^{\Lambda}, Y_{\mu}\right]=\hat{P}_{\Lambda} \psi\left[T^{\Lambda}, Y_{\mu}\right]
$$

For the resulting quantum state to be independent of the foliation condition, we need to satisfy the quantum equivalent to Equation (17), namely

$$
\left[\hat{\Pi}_{\Lambda}+\hat{P}_{\Lambda}, \hat{\Pi}_{\Sigma}+\hat{P}_{\Sigma}\right]=0
$$

on whatever the Hilbert space for the theory would be, as discussed by Kuchar [9]. If this condition holds, then the Unruh formalism itself provides the basis for a quantum theory of black holes that is foliation and gauge invariant. However, the hypertime formalism allows us to use any foliation that can be specified by our embedding variables. This can be seen to be important in the case of the Unruh foliation, by the following problem: In that foliation, the spacelike hypersurfaces avoid the apparent horizon, as discovered by Hajicek 13, 15. This makes that approach inappropriate for the study of the internal dynamics of black hole, and of Hawking radiation. The hypertime variables, however, allow us to work on foliations that extend into the black hole, through the apparent horizon.

One feature of this calculation is that we have discovered the importance of the mass function $m(r)$ in the quantum theory of spherically symmetric spacetimes. The mass function is central to understanding the semiclassical stability of the inner horizon in black holes, as has been discovered by Hiscock [8], Israel and Poisson [18]. It may be possible that this formalism will therefore provide some insight into that problem, and maybe even initial insight into a full quantum version of the problem. Furthemore, the divergent behaviour of $m(r)$ found by the above authors could be important to the actual quantum state of the black hole or wormhole.

\section{Conclusion}

We have developed a hypertime geometrodynamics for spherically symmetric black holes and wormholes, as well as collapsing matter field systems. The formalism is constructed around the effective ADM mass function, and the embedding variables are suited to work on the apparent horizons of wormholes and black holes. The approach seems apt as a starting 
point for the quantization of realistic black holes, via a hypertime functional Schrödinger equation.

\section{Acknowledgments}

I would like to sincerely thank Bill Unruh for insightful comments on the boundary constraint problem, Cynthia Foo for support, and Malcolm MacCallum and Queen Mary and Westfield College, London, for hospitality. The major part of this work was performed at the University of British Columbia and was supported by the Natural Sciences and Engineering Research Council of Canada.

\section{References}

[1] P. A. M. Dirac, Phys. Rev. 114, 924 (1959).

[2] R. Arnowit, S. Deser and C. W. Misner, Gravitation: An Introduction to Current Research, ed. L. Witten (Wiley, New York, 1962).

[3] C. J. Isham and K. V. Kuchař, Annals of Physics 164, 288 (1985).

[4] C. J. Isham and K. V. Kuchař, Annals of Physics 164316 (1985).

[5] S. P. Braham, J. Math. Phys. 34, 115 (1993).

[6] S. P. Braham, Phys. Rev. D 49, 5606 (1994).

[7] W. G. Unruh, Phys. Rev. D 14, 870 (1976).

[8] W. A. Hiscock, Physics Letters 83A, 110 (1981).

[9] K. V. Kuchař, J. Math. Phys. 13, 768 (1972).

[10] K. V. Kuchař, "Geometrodynamics of Schwarzschild Black Holes", Utah Preprint (1994).

[11] B. K. Berger, D. M. Chitre, V. E. Moncrief, and Y. Nutku, Phys. Rev. D 8, 3247 (1973).

[12] P. Thomi, B. Isaac, and P. Hajicek, Phys. Rev. D 30, 1168 (1984).

[13] P. Hajicek, Phys. Rev. D 30, 1178 (1984).

[14] P. Hajicek, Phys. Rev. D 30, 1185 (1984).

[15] P. Hajicek, Phys. Rev. D 31, 785 (1985). 
[16] D. Louis-Martinez, J. Gegenberg, and G. Kunstatter, "Exact Dirac Quantization of all 2-D Dilaton Gravity Theories", University of Winipeg Report WIN-93-06 (1993).

[17] K. V. Kuchař, Phys. Rev. D 4, 955 (1971).

[18] E. Poisson and W. Israel, Phys. Rev. D 41, 1796 (1990). 